\title{
Surface-engineered Sensors: Polymer-based Sensors for the Capacitive Detection of Organic Pollutants in Water
}

\author{
Judith Staginus ${ }^{1}$, Isabelle M. Aerts ${ }^{1}$, Zu-yao Chang ${ }^{2}$, Gerard C.M. Meijer ${ }^{2}$, Louis C.P.M. de Smet ${ }^{1, *}$, \\ Ernst J.R. Sudhölter ${ }^{1}$ \\ 1) Department of Chemical Engineering, Delft University of Technology, Julianalaan 136, 2628 BL \\ Delft, the Netherlands \\ * Corresponding e-mail address: I.c.p.m.desmet@tudelft.nl \\ 2) Department of Electrical Engineering, Delft University of Technology, Mekelweg 4, 2628 CD Delft, \\ the Netherlands
}

\begin{abstract}
:
The matured technology of electronic noses and tongues makes it promising to utilize the technique of combining chemically diverse sensor multi-arrays with multi-component analysis tools and pattern recognition techniques for a wider range of medical and environmental applications. With a selfdesigned sensor system and flow cell setup-up we studied the capacitive response of a polydimethyl siloxane (PDMS) covered interdigitated electrode (IDE) sensor upon exposure to methyl tert-butyl ether (MtBE) contaminated MilliQ water and plain mineral water. The sensor responded to MtBE concentrations as low as $2 \mathrm{ppm}$ for both aqueous solutions with full reversibility. Signal drifts due to temperature effects can be remedied by sensor calibration. State-of-the-art sensor interfaces, system minimization and integration, and smart sensing layer design promise further significant optimization possibilities for system sensitivity, stability and response time.
\end{abstract}

Key words: electronic nose and tongue, interdigitated electrodes, polydimethyl siloxane, methyl-tertbutyl ether, drinking water standards

\section{Introduction}

Water quality remains an issue as the presence of diverse pollutants poses risks to the health of humans, flora, and fauna. Much effort is done to avoid exposure to pollution though its prevention stays priority without doubts. To minimize the uptake of water pollutants, technologies are developed to treat drinking water sources and to monitor its quality. A wellknown problem is the pollution of ground water by methyl tert-butyl ether (MtBE) nearby gas stations. MtBE is used as an anti-knock agent in gasoline and is introduced into the environment by spilling of gasoline and its leakage from underground tanks. Due to its polar ether group MtBE has a lower octanolwater partition coefficient than other organic constituents of gasoline such as toluene and benzene and therefore it migrates easily with the ground water and may eventually end up in drinking water to which it gives an objectionable taste and odor. Therefore, the drinking water standard for MtBE is set low as $20-40$ ppb by the World Health Organization (WHO) [1]. Several sensor systems can already detect and distinguish between different volatile organic pollutants. Some make use of polymer-based resistors, cantilevers and silicon disks as transducer elements [2-4] or even use the advantages of different transducers (interdigitated electrodes, cantilevers, and thermoelectric calorimeters) in a single chip design [5]. Detection of methyl tert-butyl ether (MtBE) in water at concentrations as low as in drinking water standards was realized by using commercial metal oxide sensors [6]. Others imply analytical lab techniques combined with micro-extraction technology [7]. However, these systems rely on an indirect detection method by measuring in the gas phase that is in equilibrium with the aqueous pollutant solution.

Our research goal is to study the feasibility of polymer-based interdigitated electrode (IDE) sensors for the detection of both volatiles as MtBE and non-volatile water pollutants at standard drinking water concentration directly in the water phase. This study focuses on the application of polydimethyl siloxane (PDMS) as an affinity layer for the detection of MtBE. 


\section{Theoretical Background}

Polymer-based IDE chips (Fig. 1, left) as capacitive sensor platforms for the detection of organic volatiles in the gaseous phase have already been studied intensively by Igreja and Dias. [8-10]. They discussed how the different processes of pollutant adsorption, absorption and consequent swelling of the polymer layer contribute to the overall sensor response as well as the effects of different sensor designs. For thick polymer layers - (thicknesses larger than at least one-half spatial wavelength $(\lambda)$ ) and fixed electrode geometries (unchanged electrode width $(W)$ and area $(A)$ and electrode gap $(G)$ or distance $(d)$ ) (Fig. 1, left) the changes in capacitance $(\Delta C)$ can be attributed to the change in the permittivity $(\Delta \varepsilon)$ of the sensing layer upon pollutant absorption [9] as according to the simplified eq. (1):

$\Delta C=\Delta \varepsilon \frac{A}{d}$

Direct capacitive measurements in the aqueous phase are much more complex as compared to gas-phase analysis due to the presence of ions. lons may distort the electric bending field established between the electrode fingers and with increasing ionic strength the conductivity of water increases. Adverse electrical effects on capacitance measurements have been reported [11-12]. Depending on the environmental conditions, the $\mathrm{pH}$ and redox-potential will vary and so the pollutants' speciation. But also, the polymer layer itself may retain chemical groups -such as silanol (-SiOH) in the case of PDMSon its surface and in the bulk that may ionize when exposed to liquid water. For a given polymer layer its affinity for a pollutant is predominantly determined by the relative polarity of the pollutant. A measure of polarity is the octanol-water partition coefficient, log $K_{\mathrm{ow}}$, which is $\sim 1.24$ for MtBE (Fig. 1, right) [1].
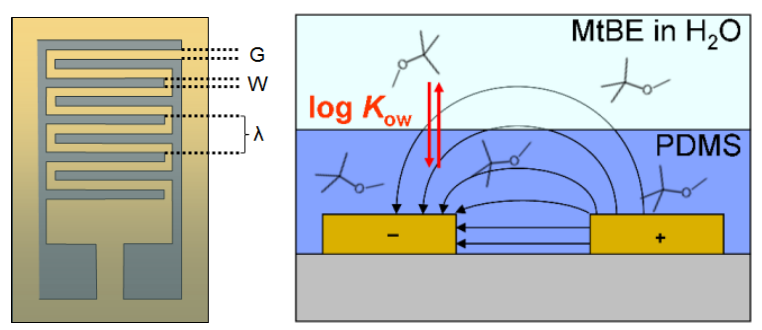

Fig. 1. Schematic representations (not to scale) of (left) a polymer-based interdigitated electrode (IDE) sensor (top view) with indication of electrode width $(W)$, electrode gap $(G)$, and spatial wavelength $(\lambda)$ and (right) the distribution of MtBE over the water phase and the PDMS layer and of (undistorted) field lines (black arrows) between the electrodes. The red arrows refer to the partition of MtBE, while log Kow stands for the logarithm of the partition coefficient.

\section{Experimental Section}

IDE Sensor Platform: The sensor chip consists of a transducer element of 221 gold IDE finger electrodes of $40 \mathrm{~nm}$ thickness on 10 $\mathrm{nm}$ thick chromium adhesion layer deposited onto borosilicate glass wafer chips of $1 \mathrm{~cm} \times 1$ $\mathrm{cm}$. The electrode finger width and gap are 6 $\mu \mathrm{m}$ and $3 \mu \mathrm{m}$, respectively. The electrode finger length is $2 \mathrm{~mm}$ (custom-made by LioniX BV). As a sensing layer served a spin-coated PDMS layer (Sylgard®184) of $\sim 35 \mu \mathrm{m}$ thickness, which is about twice the electrode wavelength of 18 $\mu \mathrm{m}$ to ensure that swelling and adsorption effects on the overall capacitance could be excluded. The sensor chip is wire-bonded to a printed circuit board (PCB) to make easy contacts to the electronics. For electronic readout served a LabView supported Universal Transducer Interface (UTI) that supplies a $5 \mathrm{~V}$ signal at $30 \mathrm{kHz}$ frequency [13].

Drop Evaporation Experiment: Drops $(4 \mu \mathrm{l})$ of different volatile organic compounds (VOCs) are deposited onto the polymer based IDE sensor. The VOCs chosen for this experiment were chloroform, MtBE, m-xylene, toluene, benzene, and $n$-hexane to cover a wide range of dielectric values. The change in capacitance was recorded from the moment of deposition until complete evaporation of the compounds.

Flow Cell Experiments: A sensor and flow cell setup was designed to allow measurements in water under continuous flow conditions while controlling the water flow rate and composition at any time. The sensor setup consists of a Teflon flow cell that is mounted onto the polymer-coated sensor chip and placed in a grounded flow cell holder from which electrical connection is made to the UTI. Solutions were delivered to the flow cell by a Harvard Syringe Pump 33 that is equipped with two individual syringe drives. One syringe was filled with MilliQ water or plain mineral water and the other one with a $10 \mathrm{mM}$ MtBE solution of the same water source. The water composition was controlled via flow rate setting. Three measurements series were performed, in which the MtBE concentration was decreased from 1 $\mathrm{mM}$ to $0.1 \mathrm{mM}$. The total flow rate was kept at $600 \mu \mathrm{l} / \mathrm{min}$.

\section{Results and Discussion}

The drop test results are shown in Fig. 2 and Tab. 1 summarizes common values of related properties of the compounds deposited including their relative dielectric constants $\left(\varepsilon_{r}\right)$, octanol-water partition coefficients (log $K_{\text {ow }}$ ) and vapor pressure as a measure for volatility. All observed changes in capacitance are in accordance with expectation from their physical properties. Capacity increases for the more 
hydrophilic (polar) compounds chloroform and $\mathrm{MtBE}$, and decreases for the more hydrophobic compounds.

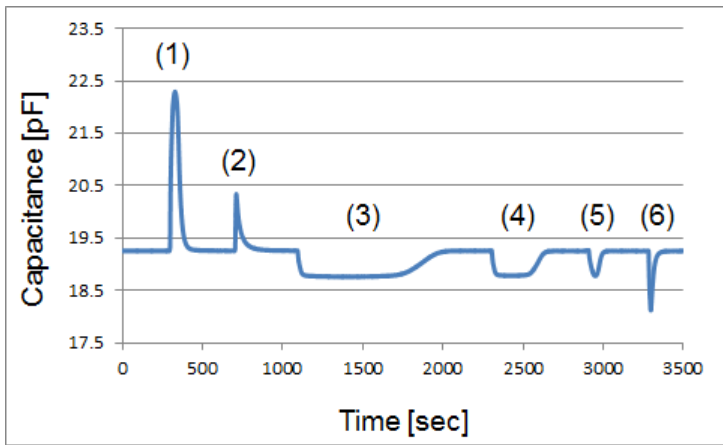

Fig. 2. Capacitive sensor response to the deposition and subsequent evaporation of the organic liquids chloroform (1), MtBE (2), m-xylene (3), toluene (4), benzene (5), and n-hexane (6).

Tab. 1: Relative dielectric constant ( $\left.\varepsilon_{r}\right)$, octanol-water partition coefficient (log Kow), and vapor pressure of the organic compounds used in this study.

\begin{tabular}{|l|c|c|c|}
\hline $\begin{array}{c}\text { Chemical } \\
\text { compound }\end{array}$ & $\begin{array}{c}\text { Relative } \\
\text { dielectric } \\
\text { constant }\left(\varepsilon_{r}\right)\end{array}$ & $\begin{array}{c}\text { Partition } \\
\text { coefficient } \\
\left(\log K_{\text {ow }}\right)\end{array}$ & $\begin{array}{c}\text { Vapor } \\
\text { pressure } \\
\text { [mmHg] }\end{array}$ \\
\hline (1) Chloroform & 4.81 & 1.97 & 197 \\
\hline (2) MtBE & 2.60 & 1.24 & 249 \\
\hline (3) m-Xylene & 2.36 & 3.20 & 8 \\
\hline (4) Toluene & 2.38 & 2.73 & 18 \\
\hline (5) Benzene & 2.28 & 2.13 & 95 \\
\hline (6) n-Hexane & 1.89 & 4.00 & 153 \\
\hline
\end{tabular}

Though the dielectric constant of Sylgard $₫ 184$ is reported to be 2.68 (at $100 \mathrm{kHz}$ ) [14], our results indicate that for thin layers, the dielectric constant is slightly lower, between 2.60 (MtBE) and 2.36 (m-xylene), since in that range the capacity response passes zero. This deviation could be related to the difference between electronic properties of thin layer PDMS and bulk PDMS as observed by others [15]. From the results it is also obvious that higher vapour pressures result in shorter retention times. With these results we have demonstrated the sensor platforms suitability for studying various physical processes.

The results of the continuous flow experiment of varying MtBE concentrations in MilliQ and plain mineral water are shown in Fig. 3 and Fig. 4. No significant effect was observed using MilliQ or plain mineral water. The conductivities of MilliQ water and the plain mineral water were measured to be $0.75 \mu \mathrm{S} / \mathrm{cm}$ and $415 \mu \mathrm{S} / \mathrm{cm}$, respectively. The effect of (small) temperature changes was much more significant. In a separate experiment it was found that the sensor has a positive temperature coefficient of $13 \mathrm{fF}$ per degree Celsius. Therefore, as done with most sensor systems correction for temperature induced change is necessary.

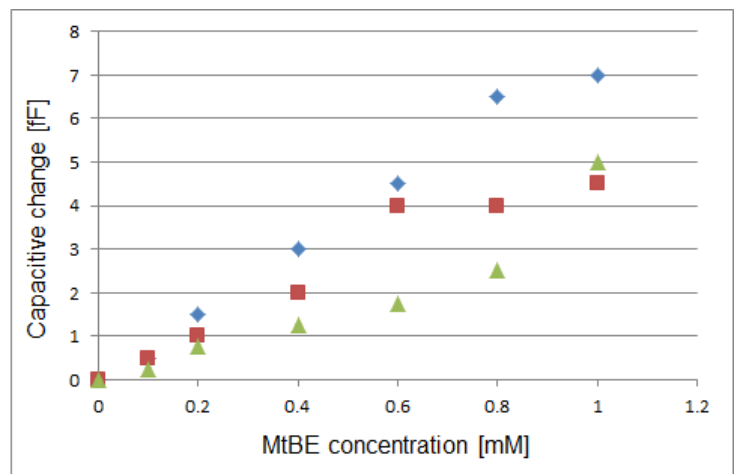

Fig 3. Changes in the average capacitance as function of the MtBE concentrations in MilliQ water. Three measuerement series were performd as indicated by the colored symbols.

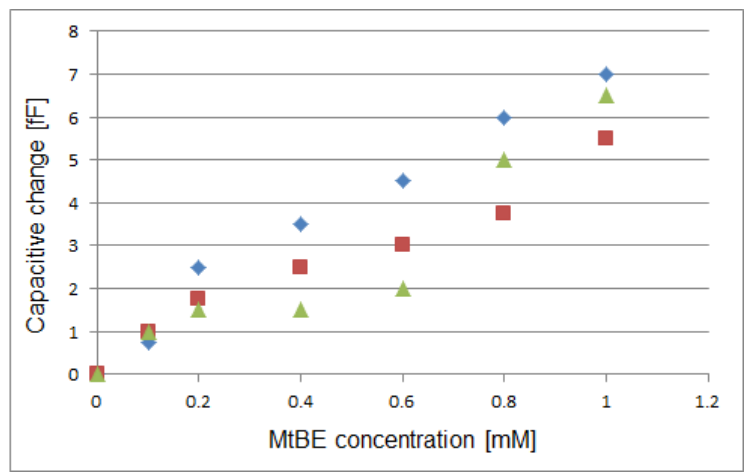

Fig 4. Changes in average capacitance as function of the MtBE concentration in plain mineral water. Three measurement series were performed as indicated by the colored symbols.

Fig. 5 shows an example of the sensor response upon ad-/absorption and desorption of MtBE (0.6 mM in MilliQ water) into the polymer layer showing complete reversibility of the sensor response.

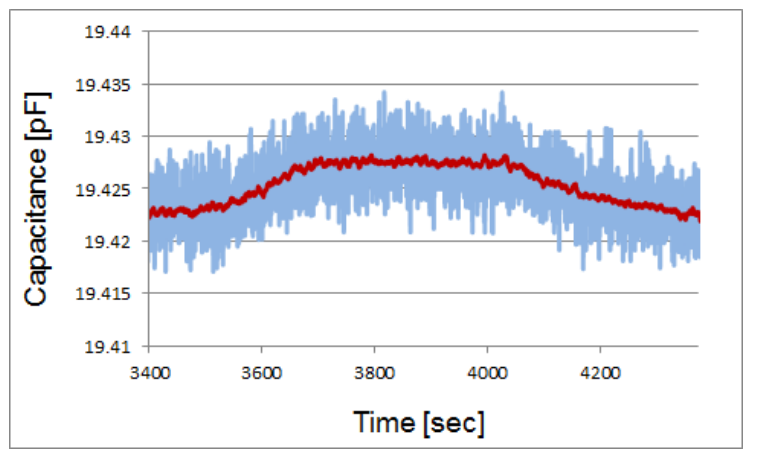

Fig 5. Changes in the capacitance averaged over 100 measurement points (red line) and the real-time value (blue line) upon exposure of a $0.6 \mathrm{mM} \mathrm{MtBE}$ solution as function of time.

It is observed in Fig. 5 that for the MtBE/PDMS system the process of ad-/absorption is faster than the desorption process. Further investigations on how other possible physical processes govern the overall sensor response is currently under progress. 


\section{Conclusion}

The results demonstrate that MtBE concentrations as low as $2 \mathrm{ppm}$ (i.e. $0.1 \mathrm{mM}$ ) could be detected successfully in water directly under both lab and environmental conditions by the designed sensor system, which utilizes a polymer-based capacitive IDE sensor platform. The target concentration to be detected lays with 20 ppb still a factor of 100 lower. However, from an electronic point of view new sensor chip technologies that will boost the sensitivity and accuracy of the UTI combined with the optimal design of both the capacitive polymerbased sensor platform including minimization and integration of electronics and sensor elements leave still much promising space for system improvements. From a chemical point of view we believe that the polymer affinity for specific pollutants can be significantly increased by incorporation of an appropriate complexation agent thereby further improving the sensor sensitivity. Also, it is anticipated that the sensor stability can be increased by integration of functional monolayers between the electrode structure and the polymer layer providing both adhesive and insulating properties.

\section{Acknowledgements}

We would like to thank Thomas Lambricht and Jochen Rutz for contributing their great ideas to this project as well as all technicians at TU Delft who were involved in the fabrication and optimization phase of the sensor setup. Special thanks go to Bart Boshuizen for LabView support and the workshop team at ChemE. We finally thank Dr. Henk Miedema and Wetsus centre of excellence for sustainable water technology for supporting and sponsoring this project.

\section{References}

[1] Methyl tertiary-ButhylEther (MTBE) in drinkingwater, Background document for development of WHO Guidelines for Drinking-water Quality (2005)WHO/SDE/WSH/05.08/122

[2] C.K. Ho, L.K. McGrath, C.E. Davis, M.L. Thomas, J. L Wright, A.S. Kooser, R.C. Hughes, Sandia National Laboratories, SAND-Report, SAND2003-3410 (2003)

[3] S. Rösler, R. Lucklum, R. Borngräber, J. Hartmann, P. Hauptmann, Sensor System for the Detection of Organic Pollutants by Thickness Shear Mode Resonators, Sensors and Actuators $B, 48,415-424$ (1998); doi:10.1016/S0925-4005(98)00079-3

[4] O. Brand, B. Mizaikoff, Membrane-based Sensor measures Pollutants present in Aqueous and Gaseous Environments, Membrane Technology, 1, 9-10 (2008); doi: 10.1016/S09582118(08)70019-3
[5] C. Hagleitner, A. Hierlemann, D. Lange, A Kummer, N. Kerness, O. Brand, H. Baltes, Smart Single-chip Gas Sensor Microsystem, Nature 414, 293-296 (2001); doi: 10.1038/35104535

[6] B.P.J. de Lacy Costello, P.S. Sivanand, N.M. Ratcliffe, D.M. Reynolds, The Rapid Detection of Methyl tert-Butyl Ether (MtBE) in Water using a Prototype Gas Sensor System, Water Science \& Technology 52 (8), 117-123, IWA Publishing (2005)

[7] S. Nakamura, S. Daishima, Simultaneous Determination of 22 Volatile Organic Compounds Methy tert-Butyl Ether, 1,4-Dioxane, 2Methylisoborneol and Geosmin in Water by Headspace Solid Phase Microextraction-gas Chromatography-Mass Spectrometry, Analytica Chimica Acta 548, 79-85 (2005); doi: 10.1016/j.aca.2005.05.077

[8] R. Igreja, C.J. Dias, Organic Vapor Discrimination using Sorption Sensitive Chemocapacitor arrays, Materials Science Forums 514-516, 1064-1067 (2006); doi: 10.4028/www.scientific.net/MSF.514516.1064

[9] R. Igreja, C.J. Dias, Dielectric Response of Interdigital Chemocapacitors: the Role of the Sensitive Layer Thickness, Sensors and Actuators B 115, 69-78 (2006); doi: 10.1016/j.snb.2005.08.019

[10] R. Igreja, C.J. Dias, Analytical Evaluation of the Interdigital Electrodes Capacitance for a Multilayered Structure, Sensors and Actuators A 112, 291-301 (2004); doi: 10.1016/j.sna.2004.01.040

[11] Z.-Y. Chang, B. P. Iliev, J.F. de Groot, G.C.M. Meijer, Extending the Limits of a Capacitive SoilWater-Content Measurement, IEEE Transaction on Instrumentation and Measurement 56 (6), 2240-2244 (2007); doi: 10.1109/TIM.2007.908317

[12] G. Behzadi, H. Golnabi, Investigation of Conductivity Effects on Capacitance Measurements of Water Liquids Using a Cylindrical Capacitive Sensor, Journal of Applied Sciences 10 (4), 261-268 (2010); doi: 10.3923/jas.2012.261.268

[13] F.M.L. Van der Goes, Low-Cost Smart Sensor Interfacing, Ph.D. Thesis, TU Delft, The Netherlands (1996)

[14] Dow Corning, Product Data Sheets on Sylgard®184 Sylicone Elastomer; downloaded from www.dowcorning.com, website last visited in 2012

[15] J. Sun, S.K. Vajandar, D. Xu, Y. Kang, G. Hu, Do. $\mathrm{Li}$, De. Li, Experimental Characterization of Electrical Current Leakage in Poly(dimethylsiloxane) Microfluidic Devices, Microfluid Nanofluid 6, 589-598 (2009); doi: 10.1007/s10404-008-0331-3 